\title{
NEW CCD OBSERVATIONS OF ECLIPSING BINARY VW CEPHEI
}

\author{
ADBDULRAHMAN ALI S. MALAWI \\ Astronomy Dept., Faculty of Science, King Abdul Aziz University
}

New CCD observations of the eclipsing binary VW Cephei were made on the 9th and 10th of October 1996 and on the 11 th of Nov. 1996. The light curves and short time-scale variations are discussed. Photometric elements were determined from analyses of the light curves. These new solutions are also discussed. 